\title{
Exercise, Protein Metabolism, and Muscle Growth
}

\author{
Kevin D. Tipton and Robert R. Wolfe
}

\begin{abstract}
Exercise has a profound effect on muscle growth, which can occur only if muscle protein synthesis exceeds muscle protein breakdown; there must be a positive muscle protein balance. Resistance exercise improves muscle protein balance, but, in the absence of food intake, the balance remains negative (i.e., catabolic). The response of muscle protein metabolism to a resistance exercise bout lasts for $24-48$ hours; thus, the interaction between protein metabolism and any meals consumed in this period will determine the impact of the diet on muscle hypertrophy. Amino acid availability is an important regulator of muscle protein metabolism. The interaction of postexercise metabolic processes and increased amino acid availability maximizes the stimulation of muscle protein synthesis and results in even greater muscle anabolism than when dietary amino acids are not present. Hormones, especially insulin and testosterone, have important roles as regulators of muscle protein synthesis and muscle hypertrophy. Following exercise, insulin has only a permissive role on muscle protein synthesis, but it appears to inhibit the increase in muscle protein breakdown. Ingestion of only small amounts of amino acids, combined with carbohydrates, can transiently increase muscle protein anabolism, but it has yet to be determined if these transient responses translate into an appreciable increase in muscle mass over a prolonged training period.
\end{abstract}

\section{Introduction}

The metabolic basis for skeletal muscle growth lies in the relationship between the rates of muscle protein synthesis and muscle protein breakdown. Muscle hypertrophy is possible only when net protein synthesis occurs: when muscle protein synthesis exceeds breakdown. It is clear that exercise has a profound effect on muscle protein metabolism, often resulting in muscle growth. However, exercise alone without certain dietary nutrients does not stimulate net muscle protein synthesis. Thus, the responses of muscle to exercise, nutrition, and hormonal status, plus the interactions among these factors must be clarified if we are to understand muscle growth.

Although the responses of muscle protein metabolism to nutrition and exercise have been studied for over a century, there are still significant gaps in our

K.D. Tipton is with the Metabolism Division of the Department of Surgery at the University of Texas Medical Branch-Galveston, Galveston, TX 77550-2720. R.R. Wolfe is with the Metabolism Division of the Departments of Surgery and Anesthesiology at the University of Texas Medical Branch-Galveston. 
understanding of the metabolic mechanisms behind exercise-induced muscle hypertrophy. The methodologies that have been used to examine these mechanisms have limited our ability to make conclusions about the response of muscle protein metabolism to nutrition and exercise. Most of the information gleaned from past studies came from indirect measures of protein metabolism such as the excretion of urea and 3-methylhistidine or from in vitro and in situ experiments. Only recently have methods become available that have enabled researchers to measure muscle protein synthesis and breakdown in humans in vivo. These methods involve the use of isotopic tracers, muscle biopsies, and, often, arteriovenous blood sampling and measurements of blood flow. Researchers now use these methods to directly measure muscle protein synthesis and muscle protein breakdown in vivo in human subjects.

In this review, we will examine the responses of muscle protein metabolism to exercise, nutrition, and hormonal status, as well as the interaction of all three in relation to muscle growth. We will primarily discuss data from reports on human subjects in vivo and utilize animal and in vitro studies as support.

\section{Effects of Exercise on Muscle Protein Metabolism}

\section{Effects of an Acute Exercise Bout on Muscle Protein Synthesis}

In recent years considerable research measuring muscle protein synthesis during and following exercise has been performed. It is now clear that an acute bout of exercise has a profound effect on muscle protein synthesis. The exact response seems to be dictated by the particular stimulus provided by the bout of exercise, namely, the type of exercise and/or the intensity at which it is performed as well as the level of training of the individual.

Resistance Exercise. Generally, it has been reported that a stimulation of protein synthesis in samples of muscle containing mixtures of various proteins (i.e., mixed muscle) results from an acute bout of resistance exercise in both animals and humans $(8,13,19-21,45,46)$. However, other studies have found no increase in muscle protein synthesis following resistance exercise $(53,62)$. The discrepant results among these studies is somewhat puzzling. It is possible that training status of the subjects may have influenced the results. A training-induced diminution of the acute response of muscle protein synthesis to resistance exercise has recently been demonstrated in both rats (19) and humans (46). The subjects in the latter studies, those in which there was no response of muscle protein synthesis to resistance exercise, were resistance-trained subjects $(53,62)$, whereas subjects in studies that reported increased muscle protein synthesis with resistance exercise were untrained $(8,45,46)$ with one exception (13). However, the intensity of the exercise routine performed by the subjects in the study reported by Chesley et al. (13) was extreme, even for well-trained individuals. It is thus likely that the challenge to the muscles was enough to stimulate muscle protein synthesis despite the training status of the subjects. Thus, it seems that muscle protein synthesis is stimulated by resistance exercise as long as the intensity of the exercise is enough to "challenge" the muscles.

Endurance Exercise. The response of mixed muscle protein synthesis to a bout of endurance exercise may not be the same as to resistance exercise. To date, 
only two studies have measured mixed-muscle protein synthesis following endurance exercise in human subjects. Carraro et al. (12) found that muscle protein synthesis in untrained volunteers was increased $\sim 25 \%$ over controls following 4 hours of walking on a treadmill. Alternatively, we were unable to find a significant increase in muscle protein synthesis in the deltoid muscles of collegiate female swimmers following an intense, interval swim workout (62). These studies suggest that training status may also play a part in the response of muscle protein synthesis to endurance exercise. Alternately, or in addition to training status, intensity of exercise may also be a determinant of the response of muscle protein synthesis to endurance exercise. The walkers exercised at a lesser intensity (12) than did the swimmers (62), and rat studies suggest that intensity of exercise affects the response of muscle protein synthesis to endurance exercise. Several authors have reported a decrease in muscle protein synthesis following intense endurance exercise in rats $(1,18,29,33)$. Furthermore, the longer the exercise bout or the more intense the exercise, the longer the rate of muscle protein synthesis is reduced. For example, Dohm et al. (18) showed that muscle protein synthesis was reduced by $71 \%$ in rats run at $28 \mathrm{~m} / \mathrm{min}$ to exhaustion, but when rats were run for only 1 hour, muscle protein synthesis was reduced by only $30 \%$. Moreover, rats that ran at a lesser intensity demonstrated no reduction of muscle protein synthesis (3). Thus, it seems that muscle protein synthesis may respond to endurance exercise in an intensity-dependent manner. The response of muscle protein synthesis to resistance and endurance exercise of various intensities is summarized in Table 1.

The fiber type of the muscles examined likely has contributed to variability in the responses of muscle protein synthesis to the various exercise bouts examined thus far. Although there have been no studies in humans that have examined the protein synthesis of different muscle fiber types in response to exercise, rat studies indicate that fast-twitch and slow-twitch muscle fibers demonstrate variable responses of protein synthesis to a particular exercise bout $(25,69,70)$. Similarly, the responses of the various protein subfractions in the mixed muscle protein may influence the overall response to an exercise bout.

Mixed muscle protein synthesis represents a weighted average of the different types of proteins in the muscle, each of which may respond differently to a particular exercise bout. Methodology is now available to examine the synthesis rates of myofibrillar, mitochondrial, and sarcoplasmic subfractions (52) as well as individual muscle proteins $(2,31)$ in humans in vivo. One might envision a scenario in

Table 1. Directional Change From Rest or Control Values of Muscle Protein Synthesis Following Exercise

Substrate
Resistance exercise

Dynamic exercise

Dyngicercise
Intensity

Response to exercise 
which myofibrillar protein synthesis is primarily responsible for the observed stimulation of mixed muscle protein synthesis by resistance exercise (66), whereas other proteins (e.g., mitochondrial proteins) more readily respond to an endurance exercise bout. However, no studies have examined the response of different muscle protein subfractions or proteins to different types of exercise or to various exercise intensities and durations. Clearly, this type of information would be very valuable in our attempt to understand how muscle protein synthesis is affected by exercise.

\section{Effects of an Acute Exercise Bout on Muscle Protein Breakdown}

When examining the mechanisms of muscle growth, it is necessary to determine the response of muscle protein breakdown as well as synthesis. There is considerably less information available concerning exercise-induced effects on muscle protein breakdown than on protein synthesis, especially with respect to endurance exercise. Early studies reported discrepant results. For example, based upon excretion of urea and 3-methylhistidine, muscle protein breakdown was reported to increase $(18,50)$, remain unchanged $(12,47)$, or decrease $(43)$ following exercise. The discrepancies among these results illustrate the difficulties in interpretation when using indirect measures of muscle protein metabolism.

More recently, two methods to study muscle protein breakdown in vivo have been developed that are based on the dilution of isotopic enrichment in the muscle intracellular protein pool due to muscle protein breakdown $(8,76)$. These methods have been utilized to measure muscle protein breakdown following heavy resistance exercise bouts in the legs of untrained volunteers $(8,45,46)$. In each of these studies, muscle protein breakdown was increased following resistance exercise but to a lesser extent than was muscle protein synthesis. Net muscle protein balance was increased but, in the absence of food intake, muscle protein breakdown exceeded synthesis, and net muscle protein balance remained negative (Figure 1).

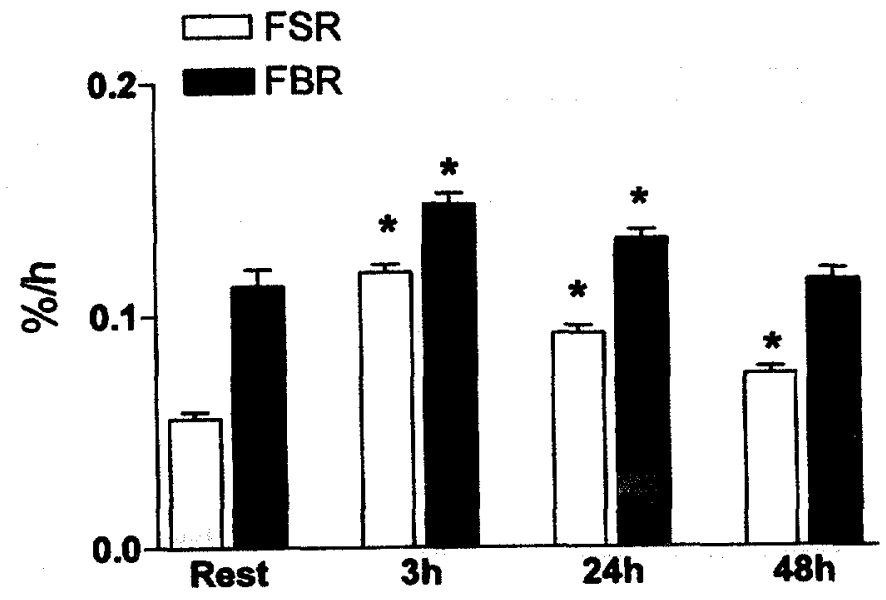

Figure 1 - Muscle protein synthesis (FSR) and breakdown (FBR) at rest and 3, 24, and 48 hours after exercise. ${ }^{*} \boldsymbol{p}<.05$ versus respective value at rest. Adapted from Phillips et al. (45). 
Table 2 Directional Change From Rest or Control Values of Muscle Protein Breakdown Following Exercise

Substrate

Resistance exercise

Dynamic exercise
Intensity

Response to exercise

\begin{tabular}{ccc} 
Resistance exercise & High & $\uparrow$ \\
& Moderate & $\leftrightarrow$ \\
Dynamic exercise & High & $\uparrow$ \\
& Moderate & $\leftrightarrow$ or $\uparrow$ \\
\hline
\end{tabular}

There are no studies in humans that have examined the response of muscle protein breakdown to endurance exercise. However, several studies utilizing rat in situ and in vitro models suggest that muscle protein breakdown is increased following both swimming and running $(3,18,34)$. Degradation of myofibrillar proteins, based on the release of 3-methylhistidine, a marker of myofibrillar protein breakdown, does not appear to contribute to the increase in the breakdown of mixed muscle protein (based on release of tyrosine) following exercise (34). These results lend support to the notion that different protein fractions may respond differently to different types of exercise bouts. The response of mixed muscle protein breakdown to exercise is summarized in Table 2.

\section{Effects of Chronic Exercise Training on Muscle Protein Metabolism}

Although most healthy, weight-stable adults are in a state of net muscle protein balance (i.e., muscle protein synthesis equals muscle protein breakdown), it is clear that chronic exercise training over a sufficient period of time may cause muscle hypertrophy. In other words, net muscle protein synthesis occurs during the training period. Common experience-for example, the fact that bodybuilders lift weights rather than run long distances-tells us that endurance exercise training will result in less muscle hypertrophy than will resistance exercise training. However, it is possible for endurance training to result in increases in muscle strength (37) and muscle fiber cross-sectional area (14). Accordingly, it seems likely that endurance exercise training has a positive impact on net muscle protein balance, at least with some proteins or protein subfractions, such as mitochondrial proteins. Unfortunately, the metabolic responses of specific muscle proteins to chronic resistance exercise have not been extensively studied, and there are no reports on the metabolic responses of specific muscle proteins to endurance exercise.

Any accretion of muscle protein as a result of training must result from a positive net protein balance. Therefore, although at any given time net muscle protein balance could be either positive or negative, the overall summation of protein balance over time must be positive.

An intriguing question is the extent to which muscle hypertrophy results from a change in the basal level of muscle protein balance. It is often suggested that hypertrophy due to resistance exercise training is caused by an increase in the basal 
level of muscle protein synthesis such that during much of the day, the muscle is in a more positive net protein balance. A series of studies concluded that resistance exercise training, even for as little as 2 weeks, increases the basal level of muscle protein synthesis (71-74), thus supporting the view that hypertrophy occurs because of an increase in the basal level of muscle protein synthesis. However, the design of these studies renders such a conclusion questionable. Although the initial pretraining measurement of muscle protein synthesis was made at rest, the final posttraining measurement was made shortly (3-24 hours) after the final bout of resistance exercise (71-74). The response of muscle protein synthesis to an acute bout of resistance exercise lasts up to 48 hours $(13,39,45)$ (Figure 1), so it is not possible to distinguish the effect of training on muscle protein synthesis from the acute effect of the final resistance exercise bout. Therefore, the increase in muscle protein synthesis attributed to resistance training may have been, at least in part, due to the previous exercise bout. Furthermore, in a cross-sectional study, the resting muscle protein synthesis and net muscle protein balance of resistance-trained subjects was no different from that of untrained subjects (46). Taken together, these studies do not provide convincing support for the notion that training-induced muscle hypertrophy is caused by an increase in the basal level of muscle protein synthesis.

Rather than being caused by an increase in the basal level of net protein balance, it is possible that muscle hypertrophy occurs because of a series of transient increases in net muscle protein balance in response to individual workouts. Accordingly, the accretion of muscle protein over the duration of a training period could be the sum of the accretion response to each exercise bout performed during that period. Consistent with this hypothesis is the fact that muscle protein synthesis and net muscle protein balance remain elevated above resting levels for up to 48 hours following an intense resistance-exercise bout $(13,39,45)$ (Figure 1). The sum of these responses repeated many times over a period of training could be expected to add up to an appreciable gain in muscle mass without an inherent change in the basal level of muscle protein synthesis. These acute responses were measured primarily in the fasted state, but muscle growth over a chronic training period would be a response to the combination of exercise and any meals consumed during the training period. We will present more detail on the interactive effect of exercise and nutrition on muscle protein synthesis and net muscle protein balance below, but it is germane to the present discussion. Rates of muscle protein synthesis and net muscle protein balance are greater when amino acid availability is increased following a resistance exercise bout than when subjects are fasted (61), and they are greater at rest when amino acid availability is increased (9). Thus, any meals consumed during the time that muscle protein metabolism is increased by each exercise bout (up to 48 hours) would presumably enhance the anabolic response to the exercise bout and contribute to muscle gain. Therefore, it seems more likely that muscle hypertrophy due to resistance exercise training is due to the accumulated set of responses to the individual exercise bouts, rather than to a change in the basal level of muscle protein synthesis.

That organ systems adapt to overload is a basic physiological principle of training. This principle is based on the idea that, as a physiological system or trait adapts to a training load, increasing intensity will be necessary for progressive adaptive increases to occur. As with most other adaptations to training, muscle hypertrophy seems to adhere to this overload principle, and this is one of the reasons that "periodization" has become a popular component of resistance training. Results 
from a cross-sectional study in resistance-trained and untrained human volunteers suggest that there is a metabolic basis for an adaptation of muscle hypertrophy due to resistance exercise. The responses of muscle protein synthesis and breakdown to a resistance exercise bout at the same relative load were both reduced in trained subjects compared to untrained subjects (46). Similarly, in rats that had been resistance trained for 8 weeks, muscle protein synthesis in response to an acute exercise bout was not increased over controls, whereas muscle protein synthesis in untrained rats subjected to the exercise bout at the same relative load was elevated over control values (19).

\section{Dietary and Hormonal Effects on Muscle Protein Metabolism}

\section{Response of Muscle Protein Metabolism to Nutritional Factors}

Nutritional substrates clearly have a dramatic effect on muscle protein metabolism and muscle growth. In the following section, we will examine the influence of nutritional substrates on muscle protein metabolism at rest. Later, we will discuss how exercise may alter this nutritional influence.

Amino Acids. Although amino acids are required precursors for muscle protein synthesis, early investigators did not acknowledge a strong relationship between amino acid availability and muscle protein synthesis (28). More recently, several authors have demonstrated that increased amino acid availability from exogenous amino acids elevates muscle protein synthesis and results in positive net muscle protein balance $(5,9,30,57)$. When results from a number of these studies are taken together, it is clear that there is a close relationship between the rate of muscle protein synthesis and the intracellular availability of amino acids (i.e., the presence of amino acids transported into the intracellular compartment from the extracellular space and amino acids derived from muscle protein breakdown). Thus, we propose the concept that intracellular amino acid availability is closely regulated. Increases in the intracellular concentration of amino acids have been demonstrated following both infusion (9) and oral administration (63) of amino acids. Changes in the intracellular availability of essential amino acids caused by changes in the rates of appearance or disappearance of amino acids will cause a coordinated response of one or more of the processes of muscle protein synthesis, breakdown, or transport in order to maintain or restore a constant amino acid concentration.

There are at least two possible, and by no means exclusive, mechanisms whereby increasing amino acid availability stimulates muscle anabolism. Perhaps the most obvious possible mechanism involves a mass-action effect from the supplied amino acids; tRNA necessary for translation of amino acids to proteins is available in muscle in amounts that exceed the available amino acids. Accordingly, increasing the amino acid availability will drive muscle protein synthesis simply by charging more tRNA. No studies of the extent of tRNA charging by amino acids and the relationship of any such charging to muscle protein synthesis have been performed, so this issue remains unresolved.

It is also possible that certain amino acids, either individual amino acids or groups of amino acids (e.g., branched chain amino acids), may signal initiation of the translation process. Rennie and colleagues (56) demonstrated that single essential 
amino acids given in a flooding dose stimulate muscle protein synthesis, whereas nonessential amino acids have no such effect. Moreover, we have recently shown that it is unnecessary to include nonessential amino acids in a solution that stimulates muscle anabolism in resting volunteers (63). These studies are consistent with the hypothesis that one or more of the essential amino acids may act as a signal for initiation of muscle protein synthesis. However, stimulation of protein synthesis through mass action cannot be ruled out based solely on this evidence. It is possible that sufficient nonessential amino acids are present and that the addition of large amounts of essential amino acids provides enough tRNA charging to drive protein synthesis.

Most of the evidence supporting the concept that amino acid availability may act as a signal to activate translation initiation comes from in vitro studies. Several reports demonstrate a rapid stimulation of initiation that is triggered by amino acids (65) or even single amino acids (35). The details of the mechanisms are beyond the scope of this review, but they have been discussed elsewhere (42). In general, proteins called eukaryotic initiation factors (eIF), in particular eIF4E, are important components of the control of the initiation of translation. When eIF4E forms a complex with eIF4G, initiation of translation is promoted. Binding of eIF4E to eIF4G is normally prevented because the eIF4E is bound to a binding protein, eIF4E-BP1, phosphorylation of which releases eIF4E. Provision of amino acids enhances this phosphorylation process, thereby allowing protein synthesis to proceed (64). In vivo evidence in humans for control of the initiation of protein synthesis by these initiation factors has yet to be elucidated, but in rats, the formation of the active eIF4E-eIF4G complex is increased in response to protein feeding $(64,75)$.

Although the relationship between intracellular amino acid availability and muscle protein breakdown is not as clear as that between amino acid availability and muscle protein synthesis, the response of muscle protein breakdown to provision of exogenous amino acids in certain situations is consistent with such a relationship. An increase in intracellular amino acids only inhibits muscle protein breakdown under certain conditions. For example, hyperaminoacidemia at rest has little or no effect on muscle protein breakdown (9). Yet, when amino acids are given following resistance exercise, when muscle protein breakdown would normally be elevated $(8,45)$, there is no increase in protein breakdown $(9,61)$. This conditional inhibition of muscle protein breakdown is consistent with the notion that the relation between intracellular availability and muscle protein breakdown occurs primarily when the intracellular pool becomes depleted. For example, if the initial response to exercise is an increase in muscle protein synthesis, the intracellular pool will be transiently decreased, activating protein breakdown to maintain the level of intracellular amino acids. Alternatively, because there are different pathways of muscle protein breakdown, it is possible that following exercise, the lysosomal pathway becomes predominant in mediating the acceleration of muscle protein breakdown (32), and this pathway may be responsive to amino acid availability.

Carbohydrates and Fats. In resting muscle the influence of energy state would seemingly be unimportant in the regulation of muscle protein anabolism. Thus, the influence of substrates other than amino acids (i.e., carbohydrates and fats) should presumably operate indirectly via metabolites of these substrates or via hormonal responses to these substrates. There is little information on the response of muscle protein metabolism to carbohydrates or lipids, per se, at rest, and there is 
scant evidence using in vitro or animal models, most likely because a rationale for such study is not as readily forthcoming as with amino acids. It was recently demonstrated that neither lipids nor carbohydrates stimulated muscle protein anabolism in the absence of amino acids (58). Thus, the only likely influence of either substrate on muscle protein metabolism is indirectly through the actions of hormones. In particular, carbohydrate ingestion may have a large influence on muscle protein metabolism by stimulating insulin secretion.

\section{Response of Muscle Protein Metabolism to Hormones}

It is difficult to characterize the effect of hormones on protein metabolism in vivo. As with all other aspects of endocrinology, the potential for interactions with other hormones and various metabolites and physiological mechanisms is great. There are several hormones that seem to have a dramatic effect on muscle protein metabolism and muscle growth. A complete overview of endocrinological effects on muscle protein metabolism is beyond the scope of this review, but we will attempt to briefly address the hormones that seem to have the most impact on muscle protein metabolism.

Insulin. The influence of insulin on muscle protein metabolism has been well studied, but it remains somewhat controversial. Shortly after insulin was discovered, it was determined that insulin treatment ameliorated the muscle wasting associated with diabetes. Yet, even today, there is no consensus on the action of insulin on in vivo muscle protein synthesis. It is possible, however, to eliminate the confusion if the results are viewed in the context of amino acid availability for protein synthesis. Systemic infusion of insulin causes a significant reduction in blood amino acid levels, thus reducing amino acid delivery to the muscles and amino acid availability for protein synthesis. When amino acid levels are not increased by ingestion or infusion during hyperinsulinemia, muscle protein synthesis is not increased (4). On the other hand, if amino acids are provided during hyperinsulinemia, muscle protein synthesis increases $(4,59)$. Moreover, muscle protein synthesis is also increased when insulin is infused locally, such that systemic amino acid levels are unaffected (7). Thus, it seems that an increase in insulin concentration increases muscle protein synthesis as long as amino acid availability is maintained.

Androgens. There has been a great deal of recent attention given to the effect of androgens on muscle growth. Common experience with many athletes as well as many recreational weightlifters tells us that testosterone and related compounds can increase muscle growth. However, the effect of testosterone on muscle protein synthesis in vivo was debated until Bhasin et al. (6) recently demonstrated unequivocally that testosterone injections in nonexercising males dramatically increase both muscle size and strength compared to placebo injections. The testosterone-induced chronic increase in muscle size is due to a substantial increase in muscle protein synthesis that is presumably controlled at the transcriptional level (24). Muscle protein breakdown is unaffected by testosterone injection so net muscle protein balance is positive. Similarly, oxandrolone, a synthetic testosterone analog, increased muscle protein synthesis without an increase in muscle protein breakdown when given orally to male subjects (54).

Androstenedione, an over-the-counter supplement, has received a great deal of recent attention due to its popularity among athletes. Oral intake of androstenedi- 
one purportedly increases testosterone levels and, consequently, muscle size and strength. Recent studies utilizing the recommended dosage of androstenedione (100 mg/day) have failed to demonstrate an increase in blood testosterone levels $(38,49)$ or, more importantly, muscle protein synthesis $(49)$. Reports of larger doses ( $300 \mathrm{mg} /$ day) have yielded equivocal results; both increased circulating testosterone concentrations (38) and no change in testosterone concentrations have been reported (36), but no indices of muscle protein synthesis were measured. Furthermore, both $100 \mathrm{mg} /$ day and $300 \mathrm{mg} /$ day resulted in increased estrogen levels (36, 38,49 ). Thus, the use of androstenedione at these doses for stimulation of muscle anabolism appears to be unwarranted.

Growth Hormone. Our understanding of the actions of growth hormone on muscle growth is incomplete, partly because of numerous interactions of growth hormone with other hormones and metabolic factors. Anabolic actions of growth hormone are presumably mediated primarily by insulin-like growth factor-I (IGFI). There is evidence that both growth hormone (27) and IGF-I (26) acutely increase muscle protein synthesis in healthy resting volunteers, whereas other studies failed to show any increase in muscle protein synthesis $(15,67)$. Additionally, long-term treatment with growth hormone had only modest effects on muscle size and strength and often caused unwanted side effects, such as carpal tunnel compression, arthralgia, and myalgia (16).

Cortisol. Hypercortisolemia has long been associated with muscle catabolism. Indeed, increased muscle protein breakdown and amino acid efflux from muscle have been attributed to cortisol $(17,55)$. Direct effects of increased cortisol on muscle protein breakdown in vivo are difficult to demonstrate in healthy humans, but such effects may be important in altered physiological states. One study demonstrated (23) that muscle protein synthesis and breakdown were not different from control values during hypercortisolemia in healthy volunteers. However, the interaction of cortisol with 2 weeks of bed rest caused a dramatic increase in protein breakdown without a concomitant increase in muscle protein synthesis; thus, increased muscle catabolism resulted from the hypercortisolemia plus long-term inactivity.

\section{Interactive Effects of Exercise, Nutrition, and Hormones on Muscle Protein Metabolism}

The impact of exercise and exercise training on muscle protein metabolism and muscle growth must be considered in the context of the interaction of the exercise with nutritional and hormonal factors that are present during a normal day. Muscle protein synthesis and breakdown are elevated for more than 24 hours after resistance exercise $(13,39,45)$ (Figure 1). Any meal that is consumed during this time could influence muscle protein metabolism. In the absence of food intake, both muscle protein synthesis and breakdown are increased in response to resistance exercise, but the rate of muscle protein breakdown exceeds synthesis such that the net muscle protein balance remains negative $(8,45,46)$. As such, muscle hypertrophy will occur only if food is ingested in the $24-48$ hours following exercise. The interactive effect of nutrition and exercise on muscle protein metabolism have only recently been investigated, so many aspects of the interactions remain to be elucidated. Most of the available information is derived from studies of resistance exercise; there are only a few animal studies using endurance exercise. 


\section{Interaction of Amino Acids and Resistance Exercise}

Amino acid availability is central to the control of muscle protein metabolism. A meal containing protein or a dietary supplement that includes amino acids or protein will increase the availability of amino acids to the muscle. We demonstrated that increasing amino acid availability by intravenous infusion following resistance exercise dramatically increased muscle protein synthesis and net muscle protein balance in healthy volunteers (9). Net muscle protein balance was changed from negative (i.e., catabolic) to positive (i.e., anabolic) as a result of the hyperaminoacidemia. Further, the anabolic effect of the amino acids following exercise was greater than at rest, suggesting that the interaction of exercise and amino acids is additive (9). Muscle protein breakdown during hyperaminoacidemia following resistance exercise was no different than resting muscle protein breakdown. Thus, the expected increase of muscle protein breakdown following exercise was ameliorated by the increased amino acid availability. These results are consistent with the hypothesis that muscle protein metabolism is regulated by the intracellular amino acid pool. Because intracellular amino acid availability was enhanced by increased delivery and inward transport of amino acids, sufficient amino acids were available for the increased muscle protein synthesis without increased muscle protein breakdown.

Intravenous infusion of amino acids is a useful method for examining the response of muscle protein metabolism to hyperaminoacidemia. However, it is not a viable method for delivering amino acids in free-living humans, and it was not clear that ingestion of amino acids would be as effective. First-pass splanchnic extraction of amino acids accounts for a large proportion of ingested amino acids (20-90\%, depending on the particular amino acid) $(40,41)$. Additionally, because exercise increases splanchnic protein degradation (68), splanchnic uptake of amino acids could be increased following exercise to compensate for amino acids lost during exercise. Consequently, we examined the response of muscle protein metabolism in human volunteers during ingestion of amino acids following resistance exercise (61). Although net muscle protein balance was negative during placebo ingestion, it was positive during ingestion of mixed amino acids as well as during ingestion of essential amino acids only. The increase in net balance was primarily a result of increased muscle protein synthesis. Clearly, amino acid availability can be increased as effectively via oral intake as with intravenous infusion; thus, any meal containing an amino acid source consumed within at least the first 24 hours after resistance exercise should result in muscle anabolism.

\section{Carbohydrates and Insulin}

The effect of consuming carbohydrates or fats on postexercise muscle protein metabolism has received only limited study. Any effect of carbohydrate ingestion on postexercise muscle protein metabolism will likely be due, at least in part, to the resulting elevation of insulin. Amino acids alone are poor insulin secretagogues, but a large carbohydrate-containing meal causes hyperinsulinemia. Elevated insulin levels at rest may increase muscle protein synthesis provided sufficient amino acids are available to support protein synthesis $(4,59)$. However, following resistance exercise, hyperinsulinemia had no effect on muscle protein synthesis, but it did prevent the normal, postexercise increase in muscle protein breakdown (10). In another study, ingestion of carbohydrates alone did not significantly increase muscle 
protein synthesis following resistance exercise in humans (53). Muscle protein breakdown was not directly measured, but 3-methylhistidine excretion, an index of myofibrillar protein breakdown, was decreased when carbohydrates were consumed following resistance exercise (53). Although the excretion of 3-methylhistidine is not a reliable measure of myofibrillar protein degradation (51), this finding is consistent with the idea that postexercise muscle protein breakdown was reduced by carbohydrate ingestion and the associated hyperinsulinemia (10). Muscle protein synthesis was stimulated in rats fed a mixed amino acid-carbohydrate meal following intense treadmill exercise, whereas muscle protein synthesis was unchanged in rats that consumed a carbohydrate meal, despite similar insulin levels in the two groups (29). Figure 2 summarizes the response of muscle protein synthesis to exercise and the interactive effects of exercise, insulin, and amino acids.

Although hyperinsulinemia does not stimulate muscle protein synthesis following resistance exercise, insulin does have a permissive role in muscle protein synthesis. Farrell and colleagues $(19,21,22)$ performed a series of studies showing that resistance exercise would not increase muscle protein synthesis without the presence of a minimal level of insulin. There seems to be a critical concentration of insulin below which muscle protein synthesis will not respond to resistance exercise. Further, it was demonstrated that the level of insulin necessary is dependent on the exercise intensity (20).

Since it is clear that amino acids stimulate postexercise muscle anabolism by increasing muscle protein synthesis and insulin blunts muscle protein breakdown, the combination of amino acids and carbohydrates (to stimulate insulin secretion) may be a potent anabolic combination. We examined the effects of ingestion of $6 \mathrm{~g}$ of essential amino acids plus $35 \mathrm{~g}$ of carbohydrates on muscle protein synthesis and breakdown (48). Bolus ingestion of the amino acid-carbohydrate solution resulted in hyperaminoacidemia and hyperinsulinemia. Net muscle protein balance was changed from negative to positive, primarily through a stimulation of muscle protein synthesis without the expected concomitant rise in muscle protein breakdown. Similar

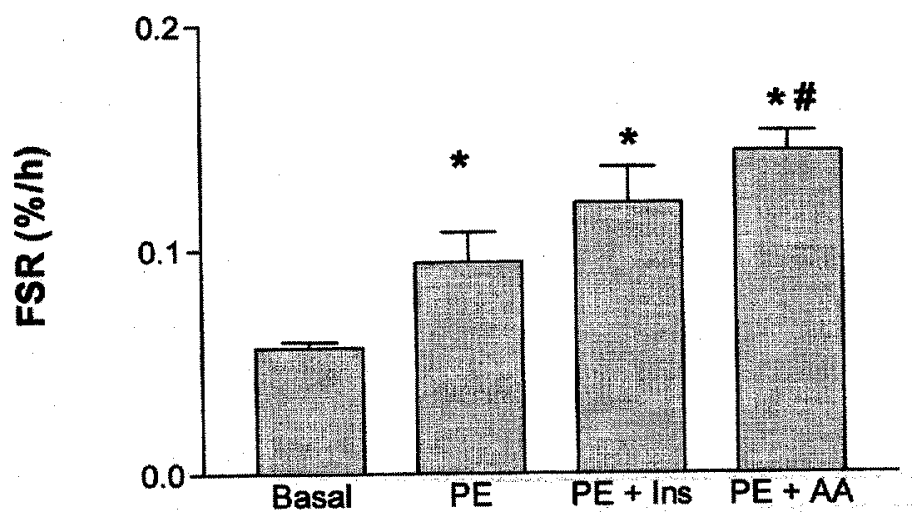

Figure 2-Muscle protein synthesis at rest (Basal), after resistance exercise (PE), after resistance exercise and during local hyperinsulinemia (PE + Ins) and after resistance exercise and during systemic hyperaminoacidemia $(\mathrm{PE}+\mathrm{AA}) .{ }^{*} p<.05$ versus basal. $\# p<.05$ versus $P E$. 
Table 3 Response of Muscle Protein Synthesis (MPS), Muscle Protein

Breakdown (MPB) and Net Muscle Protein Balance (MNB) to Nutrients Given Following Exercise

\begin{tabular}{llc}
\hline Substrate & $\bullet$ & $\begin{array}{c}\text { Response } \\
\text { following exercise }\end{array}$ \\
\hline Amino acids & MPS & $\uparrow$ \\
& MPB & $\leftrightarrow$ or $\downarrow$ \\
Amino acids + carbohydrates & MNB & $\uparrow$ from neg. to pos. \\
& MPS & $\uparrow$ \\
Carbohydrates or insulin & MPB & $\leftrightarrow$ or $\downarrow$ \\
& MNB & $\uparrow$ from negative to positive \\
& MPS & $\leftrightarrow$ or $\uparrow$ \\
& MPB & $\uparrow$ \\
& MNB & $\uparrow$ \\
\hline
\end{tabular}

Note. Responses are directional change from postexercise condition with no nutrient added.

results were reported when dogs were infused with amino acids and glucose following treadmill exercise (44). However, no comparison was made to the response of amino acids alone. The responses of muscle protein synthesis, breakdown, and net muscle protein balance to amino acids and carbohydrates are summarized in Table 3 .

It has been suggested that the stimulation of muscle protein synthesis following exercise is limited by energy availability so that any elevation in protein synthesis as a result of feeding is due to replenishment of energy. In support of this hypothesis, studies of rat muscle in vitro suggest that postexercise decreases in muscle protein synthesis are caused by diminished levels of ATP and phosphocreatine, evidence of an inhibited energy state (11). However, results of research that employed in vivo models are not consistent with this hypothesis. In one study, for example, muscle protein synthesis was not increased in rats fed a postexercise meal containing only carbohydrates, whereas rats fed an isocaloric meal of protein and carbohydrates exhibited elevated rates of synthesis. In other words, despite equivalent energy intake, muscle protein synthesis was stimulated only in rats fed protein in addition to carbohydrate (29). Accordingly, energy, per se, is unlikely to be responsible for the stimulation of muscle protein synthesis caused by meal consumption during recovery from exercise.

\section{Timing of Nutrient Ingestion}

The response of muscle protein metabolism to exercise and nutrition is also affected by the timing of nutrient ingestion in relation to the exercise bout. In a recent study, subjects ingested a solution consisting of $6 \mathrm{~g}$ of essential amino acids plus $35 \mathrm{~g}$ of carbohydrates either immediately prior to resistance exercise or immediately following the exercise (60). The response of net muscle protein balance when the drink was consumed immediately prior to exercise was considerably greater than when 


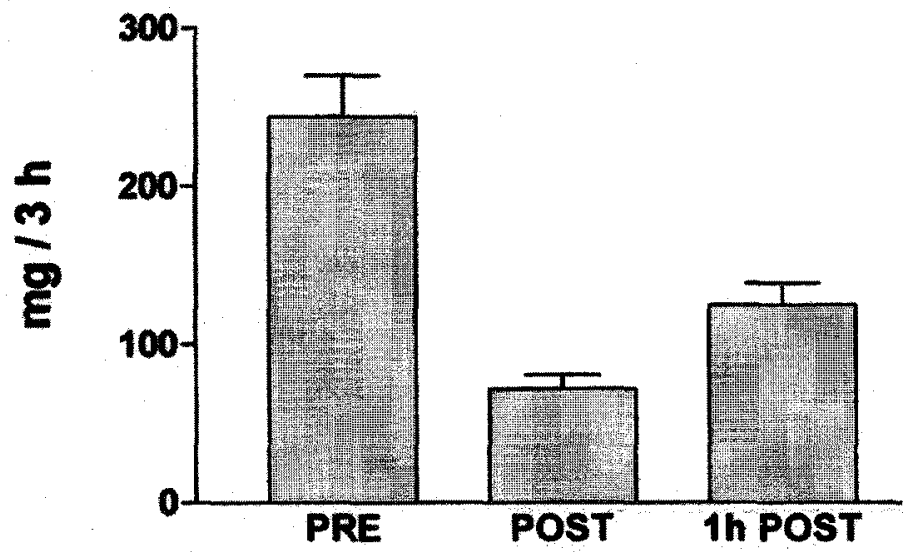

Figure 3 - Uptake of phenylalanine by muscle for 3 hours when an amino acid-carbohydrate solution is ingested immediately prior to exercise (PRE), immediately after exercise (POST), or 1 hour after exercise. All values are statistically different from one another $(p<.05)$.

the drink was consumed after exercise (Figure 3). Furthermore, the anabolic response to the mixture of amino acids and carbohydrates ingested before exercise (60) was also greater than that observed in an earlier investigation in which an identical mixture was consumed 1 hour after exercise (48). The greater anabolic response observed when the nutrients are consumed before exercise probably reflects a greater delivery (arterial amino acid concentration times blood flow) of amino acids to the muscle, thereby enabling a greater proportion of the amino acids to be taken up by the muscles $(48,60)$. Thus, ingestion of amino acids prior to exercise increases arterial amino acid concentration at a time when blood flow to the active muscles increases during the exercise bout. In contrast, blood flow to the muscles following exercise is not as great, so amino acid delivery to the muscles is comparatively less when the amino acids are consumed after exercise. The relatively lower amino acid uptake when amino acids are ingested after exercise leads to a reduced intracellular availability of amino acids for protein synthesis and therefore a smaller increase in muscle protein synthesis than when amino acids are consumed before exercise.

\section{Practical Implications}

The results of the studies described above outline the metabolic basis for muscle hypertrophy as a result of the interaction of exercise and nutrition. Although there is much still to be investigated, these studies suggest some possible practices that may be useful, not only for exercisers and athletes interested in increased fitness and performance, but also for other populations that may benefit from muscle hypertrophy, including the elderly, bedridden individuals, HIV patients, and burn patients.

The intensity of exercise may be crucial for optimal muscle hypertrophy. Although a sufficient overload stimulus is necessary to enhance muscle growth, relatively harsh exercise may inhibit the metabolic mechanisms necessary to initiate 
hypertrophy. Accordingly, light exercise may not be adequate to initiate muscle hypertrophy, whereas exercise that is too intense will reduce the desired response.

The time course of the metabolic responses of muscle proteins to exercise also has implications for recovery. Because muscle protein breakdown remains elevated for 24 hours, and muscle protein synthesis is increased for up to 48 hours $(13,39,45)$ (Figure 1), resistance training protocols that do not allow at least 48 hours of recovery between training sessions may produce less than optimal muscle development.

Although no studies have tested the influence of nutrition on the time course of muscle protein metabolism, it is likely that consumption of nutrients will impact the recovery period. Because it is apparent that elevated concentrations of circulating amino acids and insulin following exercise stimulate muscle protein synthesis and inhibit muscle protein breakdown $(8,10)$, future studies should focus on the effect of various nutrients on the time course of muscle protein metabolism.

Exercise alone (8) and the provision of amino acids alone (9) both increase rates of muscle protein synthesis; exercise alone reduces rates of muscle protein catabolism; and amino acids induce muscle anabolism. However, the most potent initiator of muscle protein synthesis is the combination of resistance exercise and elevated amino acid availability (9) (Figure 2). Moreover, because elevated levels of circulating insulin apparently inhibit muscle protein breakdown following resistance exercise, it appears that the addition of carbohydrates to a meal containing protein may increase muscle anabolism.

Insulin $(7)$ and testosterone $(6,24)$ appear to be potent anabolic hormones, but there is conflicting evidence about the influence of growth hormone and other hormones on muscle anabolism. However, the side effects associated with exogenous administration of any of these hormones preclude recommending their use by normal individuals or athletes; hormone administration should be restricted to the clinical realm.

A very small amount of amino acids ( $6 \mathrm{~g})$ plus carbohydrates ( $35 \mathrm{~g}$ of sucrose) will induce anabolism in muscle $(48,60)$, so it is tempting to interpret these findings as proof of the effectiveness of amino acid supplements in promoting muscle hypertrophy. However, there are reasons to temper such an interpretation. For example, elevations in muscle protein synthesis following ingestion of small amounts of amino acids are transient $(48,60)$; it is possible that these transient increases in anabolism add nothing to the normal anabolic responses that would result from meals ingested in the 24 hours following an appropriate resistance exercise workout. A 24-hour study of the response of muscle protein metabolism to exercise is necessary to determine the relative anabolic efficacy of amino acid supplementation compared to that induced by normal meal consumption.

Unfortunately, long-term endpoint studies of supplements are nearly impossible to control properly and may not be feasible. The major effect of exercise training and diet on muscle mass will reflect the nature and frequency of the exercise combined with normal nutrient intake. Any additional effect of a dietary supplement will be secondary and thus will be more difficult to detect over weeks of training. Calculations based on metabolic studies from our laboratory suggest that if one used typical body composition techniques to measure changes in lean body mass, it would take approximately 1 year before any effect of a dietary supplement plus resistance exercise training could be distinguished from the resistance training alone. Consequently, performance of an endpoint study of training plus dietary 
supplementation would require year-long control of each subject's diet, exercise, and other lifestyle variables before any effect of the dietary supplement was likely to be detected. However, the relatively small gains in muscle mass that may accrue from the use of dietary supplements, although difficult to detect, may be physiologically important. This potential value of dietary supplements could be especially great for individuals with reduced protein intake and/or a functional need for muscle mass gains (e.g., HIV patients, the elderly, and bedridden individuals).

The timing of supplement ingestion may be crucial as to whether muscle protein metabolism will be enhanced when compared to one's regular diet. It is clear that consumption of an amino acid-carbohydrate solution immediately prior to resistance exercise is superior to its consumption following exercise $(48,60)$ (Figure 3). Thus, any potential ability of an amino acid-carbohydrate supplement to augment muscle hypertrophy is greatly enhanced by ingestion before exercising.

\section{References}

1. Anthony, J.C., T.G. Anthony, and D.K. Layman. Leucine supplementation enhances skeletal muscle recovery in rats following exercise. J. Nutr. 129:1102-1106, 1999.

2. Balagopal, P., K.S. Nair, and W.S. Stirewalt. Isolation of myosin heavy chain from small skeletal muscle samples by preparative continuous elution gel electrophoresis: application to measurement of synthesis rate in human and animal tissue [published erratum appears in 222(2):520, 1994]. Anal. Biochem. 221:72-77, 1994.

3. Balon, T.W., A. Zorzano, J.L. Treadway, M.N. Goodman, and N.B. Ruderman. Effect of insulin on protein synthesis and degradation in skeletal muscle after exercise. Am. J. Physiol. 258:E92-E97, 1990.

4. Bennet, W.M., A.A. Connacher, C.M. Scrimgeour, R.T. Jung, and M.J. Rennie. Euglycemic hyperinsulinemia augments amino acid uptake by human leg tissues during hyperaminoacidemia. Am. J. Physiol. 259:E185-E194, 1990.

5. Bennet, W.M., A.A. Connacher, C.M. Scrimgeour, K. Smith, and M.J. Rennie. Increase in anterior tibialis muscle protein synthesis in healthy man during mixed amino acid infusion: studies of incorporation of [1-13C]leucine. Clin. Sci. 76:447454, 1989.

6. Bhasin, S., T.W. Storer, N. Berman, C. Callegari, B. Clevenger, J. Phillips, T.J. Bunnell, R. Tricker, A. Shirazi, and R. Casaburi. The effects of supraphysiologic doses of testosterone on muscle size and strength in normal men [see comments]. N. Engl. J. Med. 335:1-7, 1996.

7. Biolo, G., R.Y. Declan Fleming, and R.R. Wolfe. Physiologic hyperinsulinemia stimulates protein synthesis and enhances transport of selected amino acids in human skeletal muscle. J. Clin. Invest. 95:811-819, 1995.

8. Biolo, G., S.P. Maggi, B.D. Williams, K.D. Tipton, and R.R. Wolfe. Increased rates of muscle protein turnover and amino acid transport after resistance exercise in humans. Am. J. Physiol. 268:E514-E520, 1995.

9. Biolo, G., K.D. Tipton, S. Klein, and R.R. Wolfe. An abundant supply of amino acids enhances the metabolic effect of exercise on muscle protein. Am. J. Physiol. 273:E122E129, 1997.

10. Biolo, G., B.D. Williams, R.Y. Fleming, and R.R. Wolfe. Insulin action on muscle protein kinetics and amino acid transport during recovery after resistance exercise. Diabetes 48:949-957, 1999. 
11. Bylund-Fellenius, A.C., K.M. Ojamaa, K.E. Flaim, J.B. Li, S.J. Wassner, and L.S. Jefferson. Protein synthesis versus energy state in contracting muscles of perfused rat hindlimb. Am. J. Physiol. 246:E297-E305, 1984.

12. Carraro, F., C.A. Stuart, W.H. Hartl, J. Rosenblatt, and R.R. Wolfe. Effect of exercise and recovery on muscle protein synthesis in human subjects. Am. J. Physiol. 259:E470E476, 1990.

13. Chesley, A., J.D. MacDougall, M.A. Tarnopolsky, S.A. Atkinson, and K. Smith. Changes in human muscle protein synthesis after resistance exercise. J. Appl. Physiol. 73:1383$1388,1992$.

14. Coggan, A.R., R.J. Spina, D.S. King, M.A. Rogers, M. Brown, P.M. Nemeth, and J.O. Holloszy. Skeletal muscle adaptations to endurance training in 60- to 70-yr-old men and women. J. Appl. Physiol. 72:1780-1786, 1992.

15. Copeland, K.C., and K.S. Nair. Acute growth hormone effects on amino acid and lipid metabolism. J. Clin. Endocrinol. Metab. 78:1040-1047, 1994.

16. Cuneo, R.C. Issues regarding growth hormone replacement in growth hormone deficient adults [editorial; comment]. Aust. N.Z. J. Med. 29:289-291, 1999.

17. Darmaun, D., D.E. Matthews, andD.M. Bier.Physiological hypercortisolemia increases proteolysis, glutamine, and alanine production. Am. J. Physiol. 255:E366-E373, 1988.

18. Dohm, G.L., G.J. Kasperek, E.B. Tapscott, and G.R. Beecher. Effect of exercise on synthesis and degradation of muscle protein. Biochem. J. 188:255-262, 1980.

19. Farrell, P.A., M.J. Fedele, J. Hernandez, J.D. Fluckey, J.L. Miller, III, C.H. Lang, T.C. Vary, S.R. Kimball, and L.S. Jefferson. Hypertrophy of skeletal muscle in diabetic rats in response to chronic resistance exercise. J. Appl. Physiol. 87:1075-1082, 1999.

20. Farrell, P.A., M.J. Fedele, T.C. Vary, S.R. Kimball, and L.S. Jefferson. Effects of intensity of acute-resistance exercise on rates of protein synthesis in moderately diabetic rats. J. Appl. Physiol. 85:2291-2297, 1998.

21. Farrell, P.A., M.J. Fedele, T.C. Vary, S.R. Kimball, C.H. Lang, and L.S. Jefferson. Regulation of protein synthesis after acute resistance exercise in diabetic rats. Am. J. Physiol. 276:E721-E727, 1999.

22. Fedele, M.J., J.M. Hernandez, C.H. Lang, T.C. Vary, S.R. Kimball, L.S. Jefferson, and P.A. Farrell. Severe diabetes prohibits elevations in muscle protein synthesis after acute resistance exercise in rats [In Process Citation]. J. Appl. Physiol. 88:102-108, 2000.

23. Ferrando, A.A., C.A. Stuart, M. Sheffield-Moore, and R.R. Wolfe. Inactivity amplifies the catabolic response of skeletal muscle to cortisol.J. Clin. Endocrinol. Metab. 84:3515$3521,1999$.

24. Ferrando, A.A., K.D. Tipton, D. Doyle, S.M. Phillips, J. Cortiella, and R.R. Wolfe. Testosterone injection stimulates net protein synthesis but not tissue amino acid transport. Am. J. Physiol. 275:E864-E871, 1998.

25. Fluckey, J.D., T.C. Vary, L.S. Jefferson, and P.A. Farrell. Augmented insulin action on rates of protein synthesis after resistance exercise in rats. Am. J. Physiol. 270:E313E319, 1996.

26. Fryburg, D.A. Insulin-like growth factor I exerts growth hormone- and insulin-like actions on human muscle protein metabolism. Am. J. Physiol. 267:E331-E336, 1994.

27. Fryburg, D.A., R.A. Gelfand, and E.J. Barrett. Growth hormone acutely stimulates forearm muscle protein synthesis in normal humans. Am.J. Physiol. 260:E499-E504, 1991.

28. Garlick, P.J., and I. Grant. Amino acid infusion increases the sensitivity of muscle protein synthesis in vivo to insulin. Effect of branched-chain amino acids. Biochem. $J$. 254:579-584, 1988. 
29. Gautsch, T.A., J.C. Anthony, S.R. Kimball, G.L.Paul, D.K.Layman, and L.S. Jefferson. Availability of eIF4E regulates skeletal muscle protein synthesis during recovery from exercise. Am. J. Physiol. 274:C406-C414, 1998.

30. Gelfand, R.A., M.G. Glickman, R. Jacob, R.S. Sherwin, and R.A. DeFronzo. Removal of infused amino acids by splanchnic and leg tissues in humans. Am. J. Physiol. 250:E407E413, 1986.

31. Hasten, D.L., G.S. Morris, S. Ramanadham, and K.E. Yarasheski. Isolation of human skeletal muscle myosin heavy chain and actin for measurement of fractional synthesis rates. Am. J. Physiol. 275:E1092-E1099, 1998.

32. Kasperek, G.J., G.R. Conway, D.S. Krayeski, and J.J. Lohne. A reexamination of the effect of exercise on rate of muscle protein degradation. Am. J. Physiol. 263:E1144E1150, 1992.

33. Kasperek, G.J., and R.D. Snider. Increased protein degradation after eccentric exercise. Eur. J. Appl. Physiol. 54:30-34, 1985.

34. Kasperek, G.J., and R.D. Snider. The effect of exercise on protein turnover in isolated soleus and extensor digitorum longus muscles. Experientia 41:1399-1400, 1985.

35. Kimball, S.R., R.L. Horetsky, and L.S. Jefferson. Implication of eIF2B rather than eIF4E in the regulation of global protein synthesis by amino acids in L6 myoblasts. J. Biol. Chem. 273:30945-30953, 1998.

36. King, D.S., R.L. Sharp, M.D. Vukovich, G.A. Brown, T.A. Reifenrath, N.L. Uhl, and K.A. Parsons. Effect of oral androstenedione on serum testosterone and adaptations to resistance training in young men: a randomized controlled trial [see comments]. JAMA 281:2020-2028, 1999.

37. Kraemer, W.J., J.F. Patton, S.E. Gordon, E.A. Harman, M.R. Deschenes, K. Reynolds, R.U. Newton, N.T. Triplett, and J.E. Dziados. Compatibility of high-intensity strength and endurance training on hormonal and skeletal muscle adaptations. J. Appl. Physiol. 78:976-989, 1995.

38. Leder, B.Z., C. Longcope, D.H. Catlin, B. Ahrens, D.A. Schoenfeld, and J.S. Finkelstein. Oral androstenedione administration and serum testosterone concentrations in young men. JAMA 283:779-782, 2000.

39. MacDougall, J.D., M.J. Gibala, M.A. Tarnopolsky, J.R. MacDonald, S.A. Interisano, and K.E. Yarasheski. The time course for elevated muscle protein synthesis following heavy resistance exercise. Can. J. Appl. Physiol. 20:480-486, 1995.

40. Matthews, D.E., M.A. Marano, and R.G. Campbell. Splanchnic bed utilization of glutamine and glutamic acid in humans. Am. J. Physiol. 264:E848-E854, 1993.

41. Matthews, D.E., M.A. Marano, and R.G. Campbell. Splanchnic bed utilization of leucine and phenylalanine in humans. Am. J. Physiol. 264:E109-E118, 1993.

42. Merrick, W.C. Mechanism and regulation of eukaryotic protein synthesis. Microbiol. Rev. 56:291-315, 1992.

43. Mussini, E., L. Colombo, G. De Ponte, M. Calzi, and F. Marcucci. Effect of swimming on protein degradation: 3-methylhistidine and creatinine excretion. Biochem. Med. 34:373$375,1985$.

44. Okamura, K., T. Doi, K. Hamada, M. Sakurai, K. Matsumoto, K. Imaizumi, Y. Yoshioka, S. Shimizu, and M. Suzuki. Effect of amino acid and glucose administration during postexercise recovery on protein kinetics in dogs. Am.J.Physiol. 272:E1023-E1030, 1997.

45. Phillips, S.M., K.D. Tipton, A. Aarsland, S.E. Wolf, and R.R. Wolfe. Mixed muscle protein synthesis and breakdown after resistance exercise in humans. Am. J. Physiol. 273:E99-107, 1997. 
46. Phillips, S.M., K.D. Tipton, A.A. Ferrando, and R.R. Wolfe. Resistance training reduces the acute exercise-induced increase in muscle protein turnover. Am. J.Physiol. 276:E118E124, 1999.

47. Plante, P.D., and M.E. Houston. Effects of concentric and eccentric exercise on protein catabolism in man. Int. J. Sports Med. 5:174-178, 1984.

48. Rasmussen, B.B., K.D. Tipton, S.L. Miller, S.E. Wolf, and R.R. Wolfe. An oral essential amino acid-carbohydrate supplement enhances muscle protein anabolism after resistance exercise. J. Appl. Physiol. 88:386-392, 2000.

49. Rasmussen, B.B., E. Volpi, D.C. Gore, and R.R. Wolfe. Androstenedione does not stimulate muscle protein anabolism in young healthy men. J. Clin. Endocrinol. Metab. $85: 55-59,2000$.

50. Rennie, M.J., R.H. Edwards, S. Krywawych, C.T. Davies, D. Halliday, J.C. Waterlow, and D.J. Millward. Effect of exercise on protein turnover in man. Clin. Sci. 61:627-639, 1981.

51. Rennie, M.J., D.J. Millward, D. Halliday, and K. Lundholm. A dissenting view of the significance of 3-methylhistidine excretion in cirrhotic patients [letter]. Clin. Sci. 64:243$246,1983$.

52. Rooyackers, O.E., P. Balagopal, and K.S. Nair. Measurement of synthesis rates of specific muscle proteins using needle biopsy samples. Muscle Nerve Suppl. 5:S93-S96, 1997.

53. Roy, B.D., M.A. Tarnopolsky, J.D. MacDougall, J. Fowles, and K.E. Yarasheski. Effect of glucose supplement timing on protein metabolism after resistance training. J. Appl. Physiol. 82:1882-1888, 1997.

54. Sheffield-Moore, M., R.J. Urban, S.E. Wolf, J. Jiang, D.H. Catlin, D.N. Herndon, R.R. Wolfe, and A.A. Ferrando. Short-term oxandrolone administration stimulates net muscle protein synthesis in young men. J. Clin. Endocrinol. Metab. 84:2705-2711, 1999.

55. Simmons, P.S., J.M. Miles, J.E. Gerich, and M.W. Haymond. Increased proteolysis. An effect of increases in plasma cortisol within the physiologic range. J. Clin. Invest. 73:412$420,1984$.

56. Smith, K., N. Reynolds, S. Downie, A. Patel, and M.J. Rennie. Effects of flooding amino acids on incorporation of labeled amino acids into human muscle protein. Am. J. Physiol. 275:E73-E78, 1998.

57. Svanberg, E., A.C. Moller-Loswick, D.E. Matthews, U. Korner, M. Andersson, and K. Lundholm. Effects of amino acids on synthesis and degradation of skeletal muscle proteins in humans. Am. J. Physiol. 271:E718-E724, 1996.

58. Svanberg, E., A.C. Moller-Loswick, D.E. Matthews, U. Korner, M. Andersson, and K. Lundholm. The role of glucose, long-chain triglycerides and amino acids for promotion of amino acid balance across peripheral tissues in man. Clin. Physiol. 19:311-320, 1999.

59. Tessari, P., S. Inchiostro, G. Biolo, R. Trevisan, G. Fantin, M. C. Marescotti, E. Iori, A. Tiengo, and G. Crepaldi. Differential effects of hyperinsulinemia and hyperaminoacidemia on leucine-carbon metabolism in vivo. Evidence for distinct mechanisms in regulation of net amino acid deposition. J. Clin. Invest. 79:1062-1069, 1987.

60. Tipton, K.D., B.B. Rasmussen, S.L. Miller, S.E. Wolf, S.K. Owens-Stovall, B.E. Petrini, and R.R. Wolfe. Timing of amino acid-carbohydrate ingestion alters anabolic response of muscle to resistance exercise. Am. J. Physiol. Endocrinol. Metab. submitted: 2000.

61. Tipton, K.D., A.A. Ferrando, S.M. Phillips, D. Doyle, Jr., and R.R. Wolfe. Postexercise net protein synthesis in human muscle from orally administered amino acids. Am. J. Physiol. 276:E628-E634, 1999. 
62. Tipton, K.D., A.A. Ferrando, B.D. Williams, and R.R. Wolfe. Muscle protein metabolism in female swimmers after a combination of resistance and endurance exercise. $J$. Appl. Physiol. 81:2034-2038, 1996.

63. Tipton, K.D., B.E. Gurkin, S. Matin, and R.R. Wolfe. Nonessential amino acids are not necessary to stimulate net muscle protein synthesis in healthy volunteers. J. Nutr. Biochem. 10:89-95, 1999.

64. Vary, T.C., L.S. Jefferson, and S.R. Kimball. Amino acid-induced stimulation of translation initiation in rat skeletal muscle. Am. J. Physiol. 277:E1077-E1086, 1999.

65. Wang, X., L.E. Campbell, C.M. Miller, and C.G. Proud. Amino acid availability regulates p70 S6kinase and multiple translation factors [publishederratum appears in Biochem. J. 1998 Nov 1;335(Pt. 3):711]. Biochem. J. 334(Pt. 1):261-267, 1998.

66. Welle, S., K. Bhatt, and C.A. Thornton. Stimulation of myofibrillar synthesis by exercise is mediated by more efficient translation of mRNA. J. Appl. Physiol. 86:1220-1225, 1999.

67. Welle, S., C. Thornton, M. Statt, and B. McHenry. Growth hormone increases muscle mass and strength but does not rejuvenate myofibrillar protein synthesis in healthy subjects over 60 years old. J. Clin. Endocrinol. Metab. 81:3239-3243, 1996.

68. Williams, B.D., R.R. Wolfe, D.P. Bracy, and D.H. Wasserman. Gut proteolysis contributes essential amino acids during exercise. Am. J. Physiol. 270:E85-E90, 1996.

69. Wong, T.S., and F.W. Booth. Protein metabolism in rat gastrocnemius muscle after stimulated chronic concentric exercise. J. Appl. Physiol. 69:1709-1717, 1990.

70. Wong, T.S., and F.W. Booth. Protein metabolism in rat tibialis anterior muscle after stimulated chronic eccentric exercise. J. Appl. Physiol. 69:1718-1724, 1990.

71. Yarasheski, K.E., J.A. Campbell, K. Smith, M.J. Rennie, J.O. Holloszy, and D.M. Bier. Effect of growth hormone and resistance exercise on muscle growth in young men. Am. J. Physiol. 262:E261-E267, 1992.

72. Yarasheski, K.E., J. Pak-Loduca, D.L. Hasten, K.A. Obert, M.B. Brown, and D.R. Sinacore. Resistance exercise training increases mixed muscle protein synthesis rate in frail women and men $>/=76$ yr old. Am. J. Physiol. 277:E118-E125, 1999.

73. Yarasheski, K.E., J.J. Zachwieja, and D.M. Bier. Acute effects of resistance exercise on muscle protein synthesis rate in young and elderly men and women [published erratum appears in Am. J. Physiol. 1993 Oct;265(4 Pt. 1):following table of contents]. Am. J. Physiol. 265:E210-E214, 1993.

74. Yarasheski, K.E., J.J. Zachwieja, J.A. Campbell, and D.M. Bier. Effect of growth hormone and resistance exercise on muscle growth and strength in older men. Am. J. Physiol. 268:E268-E276, 1995.

75. Yoshizawa, F., S.R. Kimball, T.C. Vary, and L.S. Jefferson. Effect of dietary protein on translation initiation in rat skeletal muscle and liver. Am. J. Physiol. 275:E814-E820, 1998.

76. Zhang, X.J., D.L. Chinkes, Y.Sakurai, and R.R. Wolfe. An isotopic method for measurement of muscle protein fractional breakdown rate in vivo. Am. J. Physiol. 270:E759E767, 1996. 


\section{Appendix: Discussion}

SPRIET: In all of your studies, your control condition has been a totally fasted situation before and after exercise. When you were designing these studies, did you give any thought to having your control trial include feeding the individuals 2-3 hours before exercise? Was there concern you wouldn't get enough amino acids by ingestion to replicate the protein infusion situations?

TIPTON: That was one of our concerns. Also, there are limitations to the isotope experiments. For example, a steady state is required, and it is more difficult to achieve such a state in a fed condition. Moreover, many people do exercise in fasted situations. I am in the gym every morning at 6:00 a.m., and there are a lot of people working out, presumably in a fasted state, so it does have some practical applicability. I don't know if the anabolic response would be as great in the fed state. However, when we give two boluses of amino acids an hour apart, we still see an increase in the anabolic response. I suspect that there would be a jump in net muscle protein synthesis even in the fed state. What I am not convinced of is whether that increase, on the order of nanomolar changes in amino acids, translates into measurable gains in muscle mass.

SPRIET: How difficult would it be to achieve the same amino acid delivery to the muscles that you get with your amino acid supplements by ingesting normal meals?

TIPTON: I think it can be achieved through meals. Lots of athletes who hear about this conclude that amino acid supplements are great and are eager to buy some so they can "get huge." I don't know that that is a good interpretation of our data. According to some of our unpublished data with protein feedings, $I$ am convinced that $40 \mathrm{~g}$ of whey protein ultimately increases muscle protein balance to about the same extent as amino acids do; it just takes a bit longer.

GIBALA: My question is related to the traditional textbook description of the time course and mechanisms underlying changes in strength and muscle fiber hypertrophy during resistance training. Most of us probably teach our students that the initial increases in strength are due to neural adaptations, and that the fiber hypertrophy response does not occur until after several weeks of training. Has this been the consensus opinion because we haven't had methods that are sensitive enough to assess early changes in muscle protein accretion? Given the acute changes that you see in muscle protein net balance after a single bout of resistance exercise, is it possible that muscle protein anabolism may contribute to the initial increases in strength?

TIPTON: I can't prove it, but I believe that there are early increases in muscle mass that cannot currently be detected. It may take 8 weeks for this increased anabolism to be detected.

MAUGHAN: We are always told that the reason for regular feeding of protein is because the body has no non-functional protein reserve. However, if one fasts for 2 or 3 days, there is a substantial breakdown of gut protein, so there is in effect a reserve of protein that is mobilized. Do you know how quickly that mobilization occurs? Is it a matter of hours, or is it a matter of days before you begin to have those amino acids available from breaking down gut protein? 
TIPTON: I don't know. I do know that in skeletal muscle, protein breakdown occurs very quickly - within a few hours of beginning a fast-and this can provide a huge reserve of amino acids, especially for synthesis of liver proteins.

MAUGHAN: How much muscle protein do you need to break down before you see any functional impairment? There can be a substantial amount of protein breakdown in the gut with no obvious impairment in gut function, but I don't know about muscle.

TIPTON: It probably depends on the particular muscle and what that muscle is doing. In elderly individuals with sarcopenia, muscle mass is lost to a point at which muscle function can be impaired. We can measure significant loss of muscle mass in less than a week in bed-rested individuals, but $\mathrm{I}$ think it will take longer to observe noticeable impairment in strength.

COYLE: What is your opinion about whether or not the presence of insulin-like growth factors is required for muscle hypertrophy to occur?

TIPTON: It is difficult to tell from the literature how important insulin-like growth factors are for the hypertrophy of muscle. In a couple of studies that examined forearm protein balance using tracers, they did get positive effects on amino acid uptake after administration of insulin-like growth factor I, but no one has been able to replicate that result, so we are not sure. Of course, there are other hormones involved as well. Cortisol can accelerate protein breakdown, and there have been some studies suggesting that carbohydrate feedings can decrease cortisol and other stress hormone levels during exercise. Perhaps carbohydrate could be playing a role in anabolism in other indirect ways that we haven't tried to measure.

HORSWILL: The studies you've reported utilized amino acid feedings. There is evidence suggesting that differences in protein synthesis occur in response to the feeding of different dietary proteins, namely, "fast" versus "slow" proteins. Are those effects primarily due to differences in speed of protein digestion, or might they be attributed to the amino acid profiles in the different proteins?

TIPTON: The research to which you refer tested casein versus whey proteins. Casein is absorbed more slowly from the gut than is whey, so amino acids derived from casein are slower to reach a peak concentration in the blood, the peak is lower than for whey, and the concentrations of plasma amino acids derived from casein and remain relatively high in the blood for a longer time than is the case for amino acids derived from whey. My suspicion is that the casein was deemed to be better for protein synthesis because it prolonged the anabolic response. Also, they used wholebody measurements of protein synthesis and not muscle, and I don't know if the results would be the same in muscle. I suspect that there would not be a large difference between casein and whey over a 24 -hour period.

I don't think any observed difference in protein synthesis has anything to do with the amino acid profiles of the respective proteins. We found an anabolic response with only $6 \mathrm{~g}$ of essential amino acids, so I doubt that it is going to make a big difference when subjects are given $40 \mathrm{~g}$ of a protein that contains far more than $6 \mathrm{~g}$ of the essential amino acids.

EICHNER: Strength coaches ask me, "What can I give to help my athletes bulk up?" I think soon there will be a flood of new amino acid "magic potions," and each amino acid will have a myth attached to it. Ads will say, "Use tyrosine to rev up the brain, 
glutamine to rev up the lymphocytes, arginine to boost growth hormone, branched-chain amino acids to prevent central fatigue, and all of them together to drive up insulin." I think we are teaching our athletes the wrong lesson: that magic potions are important. Do you know of any evidence that any of this stuff is even equal to just insisting that the athletes eat lunch, then lift, and then eat dinner? Instead of drinking a magic potion with fragments of food, why not just teach them to exercise and eat in a healthful way?

TIPTON: I agree with you. I believe that if one were to measure 24-hour muscle protein balance and compare normal meals alone with normal meals plus amino acid supplements, any difference in protein anabolism over 24 hours would probably not be detectable. The anabolic response to the meals will probably be great enough to mask the transient response to the amino acid supplement. There might be a small difference that could show up after 2 years or so, but to do that study would be next to impossible.I think that the marketing departments of the supplement manufacturers and distributors create the popularity of most of these dietary supplements. There is really no evidence that any of these "muscle-builder" supplements works.

MURRAY: What is your understanding about the importance of the hydration status of the muscle cell and muscle protein balance? If there is a large effect, is it large enough that it becomes important to control for hydration status in proteinbalance studies?

TIPTON: I think that hydration status could possibly be important if muscle cells respond the same as liver cells - that is, if they increase protein synthesis when they swell with water and decrease protein synthesis when they shrink. Unfortunately, I don't know of any convincing evidence that these responses occur in skeletal muscle.

MURRAY: What about athletes who are laid up with injuries and muscle atrophy becomes a real concern? Is there anything in the literature with athletes or burn patients that can lead to practical advice regarding nutritional interventions designed to speed the rehabilitation process?

TIPTON: The protein metabolism of burn patients-with protein breakdown being so greatly accelerated compared to protein synthesis-is so different from the metabolism of normal individuals that burn patients are probably not good models for patients undergoing rehabilitation from other types of injuries. However, the bed rest studies probably are because with bed rest for as brief as a week one can detect a catabolic response in muscle, but this catabolism can be ameliorated with a very small amount of exercise. I' $m$ not sure about nutritional interventions, but I believe that exercise of one sort or another can be useful in minimizing the loss of muscle mass during rehabilitation.

GIBALA: Peter Hespel's group has conducted a study in which they casted subjects for two weeks to induce muscle fiber atrophy in the vastus lateralis. Subjects then received either creatine or a placebo supplement during a subsequent 10 -week rehabilitation period in conjunction with resistance training. The rate of increase in strength and muscle fiber area during rehabilitation was greater in the subjects who received the creatine supplement. Thus, creatine ingestion appeared to augment the rate of recovery following muscle disuse/atrophy.

HARGREAVES: Do you have any sense of the dose response relationship for some of the responses to administration of amino acids that you have seen? 
TIPTON: We know that amounts of amino acids as small as $6 \mathrm{~g}$ can alter the metabolic response of proteins in muscle, but we don't have information on other doses.

GIBALA: You argue that essential amino acids are superior to the non-essential ones, but as far as I can tell, this is based on only one study in which you observed no difference in net protein balance between a solution containing $40 \mathrm{~g}$ of essential amino acids and a solution containing $40 \mathrm{~g}$ of mixed amino acids. Have you actually compared non-essential amino acids against essential amino acids to specifically examine whether essential amino acids are superior for improving muscle protein net balance after an acute bout of resistance exercise?

TIPTON: We haven't done that study. The work of Mike Rennie's lab supports the hypothesis that there will be no effect on net protein balance with non-essential amino acids. When they gave boluses of non-essentials, they found no effect on protein synthesis, whereas they observed a large increase when they administered essential amino acids.

DAVIS: Is it possible to determine whether the synthesis and degradation is associated with contractile proteins rather than with proteins in connective tissues, enzymes, and so on?

TIPTON: Protein metabolism can be linked specifically to myofibrillar or sarcoplasmic or mitochondrial proteins. Even the protein metabolism in specific isomers of myosin can be examined. I can envision a scenario in which the increase in protein synthesis seen following endurance exercise is isolated more to mitochondrial proteins and less to myofibrillar proteins, with the opposite occurring after resistance exercise.

DAVIS: Is it possible that an overall gain in net protein synthesis in muscle can occur without a concomitant increase in muscle growth? In other words, could there be an increase in synthesis occurring in some proteins and an increase in degradation occurring in others so that the overall effect on muscle growth is negligible?

TIPTON: That is a very distinct possibility. So far we have studied only mixed muscle protein. We definitely know that growth occurs, so when we see increases in net muscle protein synthesis that correspond to growth, we feel a little more confident about assuming that the two phenomena are connected. But I think in the long run, different fiber types and different muscle fractions may be responding differently. 